\title{
POSITIVE-DEFINITE FUNCTIONS ON SPHERES AND SIDELNIKOV INEQUALITY
}

\author{
N. O. Kotelina And A. B. Pevnyi
}

Abstract. This article is devoted to the new proof of V. M. Sidelnikov inequality (1974). The proof is based on the theory of positive-definite functions on spheres introduced and studied by I. Schoenberg (1942).

Mathematics subject classification (2010): 42A82. inequality.

Keywords and phrases: Positive-definite functions on spheres, Gegenbauer polynomials, Sidelnikov

\section{REFERENCES}

[1] J. M. Goethals, J. J. Seidel, Spherical designs, Proc. Symp. Pure Math. A.M.S., 34, (1979), $255-272$.

[2] N. O. Kotelina, A. B. Pevnyi, Extremal properties of spherical semidesigns, St. Petersburg Math. J., 22, 5 (2011), 795-801.

[3] N. O. Kotelina, A. B. Pevnyi, Sidelnikov inequality, St. Petersburg Math. J., 26, 2 (2015), $351-$ 356.

[4] I. J. Schoenberg, Metric spaces and positive definite functions, Trans. Amer. Math. Soc., 44 (1938), $522-536$.

[5] I. J. Schoenberg, Positive definite functions on spheres, Duke Math. J., 9, 1 (1942), 96-108.

[6] V. M. SIDEL'NIKOV, New bounds for densest packing of spheres in nn-dimensional Euclidean space, Math. USSR-Sb., 24:1 (1974), 147-157.

[7] B. Venkov, Réseaux et designs sphériques, Réseaux Euclidiens, Designs sphériques et Formes Modulaires, L’Enseignement mathématique Monograph, Géneve, 37 (2001), 10-86. 